Meta

Journal des traducteurs

Translators' Journal

\title{
L'emploi de " en » et « de » dans les locutions de spécialisation
}

\section{Bruno Couture}

Volume 17, numéro 4, décembre 1972

URI : https://id.erudit.org/iderudit/002430ar

DOI : https://doi.org/10.7202/002430ar

Aller au sommaire du numéro

Éditeur(s)

Les Presses de l'Université de Montréal

ISSN

0026-0452 (imprimé)

1492-1421 (numérique)

Découvrir la revue

Citer cet article

Couture, B. (1972). L'emploi de « en " et « de " dans les locutions de spécialisation. Meta, 17(4), 227-233. https://doi.org/10.7202/002430ar d'utilisation que vous pouvez consulter en ligne.

https://apropos.erudit.org/fr/usagers/politique-dutilisation/ 


\section{BIBLIOGRAPHIE}

Craig, Robert L. et Lester R. Bittel (éd.), Training and Development Handbook, New York, McGraw-Hill Book Company, 1967, 650 p.

Fuxppo, Edwin B., Principles of Personnel Management, New York, McGraw-Hill Book Company, 1971, 585 p.

Maynard, H. B. (éd.), Handbook of Business Administration, New York, McGraw-Hill Book Company, $1968,2048 \mathrm{p}$.

Yoder, Dale, Personnel Management and Industrial Relations, Englewood Cliffs (N.J.), Prentice-Hall Inc., 1962, 667 p.

Aubert-Krier, Jane, Gestion de l'entreprise, Paris, P.U.F., Sciences économiques, "Thémis ", t. II : Activités et politiques, $1971,572 \mathrm{p}$.

JARDILlIER, Pierre, Organisation humaine des entreprises, Paris, P.U.F., "Collection du travail humain », $1965,429 \mathrm{p}$.

Marsonneuve, Jean, Dynamique des groupes, Paris, P.U.F., "Que sais-je ? ", 1969, n 1306, $128 \mathrm{p}$.

TEZEnas, J., Dictionnaire de l'organisation et de la gestion, Paris, Les Editions de l'Organisation, $1971,270 \mathrm{p}$.

\section{L'EMPLOI DE "EN" ET “DE” DANS LES LOCUTIONS DE SPÉCIALISATION}

La manière dont on utilise les prépositions révèle la connaissance qu'on a des mécanismes d'une langue. Cette constatation banale prend une signification particulière dans une situation de bilinguisme où, par suite de l'interpénétration des idiomes en présence, les locuteurs vivent dans ce sentiment, bien connu des Canadiens français, qu'on a appelé l'insécurité langagière.

Ainsi peut-on se demander si les locutions analyste en systèmes et méthodes, animateur en loisir, dessinateur en mécanique et professeur en géographie ${ }^{1}$ sont correctement formées. Disons tout de suite qu'il serait difficile de répondre globalement à cette question, car on ne tarde pas à constater, à l'examen, qu'il $\overline{1 . ~ G l a n e ́ e s ~ d a n s ~ l e s ~ a n n o n c e s ~ d e s ~ q u o t i d i e n s ~ l a ~ P r e s s e ~ e t ~ l e ~ D e v o i r, ~ l e s ~} 8$ avril et 9 août 1972. 
n'existe guère de règles générales, mais plutôt un usage propre à chaque cas. Comme il n'est pas indifférent pour le traducteur de connaître cet usage, si capricieux soit-il, et d'en déceler l'éventuelle évolution, nous allons comparer ci-après certains syntagmes de ce genre.

Il faut d'abord distinguer plusieurs sortes de locutions marquant la spécialisation, à en juger par les exemples qu'apporte le grand dictionnaire Robert, à l'article en : récolte en blé; cinq hectares plantés en vigne; dépense en électricité; être fort en sciences, sérieux en affaires, timide en amour; le premier en date, etc. ${ }^{2}$ Le sujet étant vaste, nous n'étudierons ici que trois cas, ceux de la spécialisation des études, du grade et de la profession, pour lesquels le Grand Larousse encyclopédique cite, entre autres, les exemples suivants : licencié en droit, docteur en médecine, peintre en bâtiment ${ }^{3}$.

En ce qui concerne la spécialisation de la discipline étudiée, la série des Guides de l'étudiant des P.U.F. utilise, outre l'apposition (étudiant helléniste, latiniste, angliciste, germaniste), les expressions étudiant en géographie, en sociologie, en histoire ancienne, en littérature française, etc. D'autre part, le grand dictionnaire Robert mentionne étudiant en Droit, en Sciences, en Lettres, en Médecine, en Pharmacie, mais aussi étudiant des Beaux-Arts qui, cependant, fait plutôt penser à l'établissement d'enseignement qu'à la matière étudiée. Quant au groupe étudiant de traduction ${ }^{4}$, il paraît avoir subi la contagion des locutions construites avec le mot études : études de droit, de médecine, de sciences, de piano, de chant, d'art dramatique (grand dictionnaire Robert). Il y a pourtant lieu de signaler ici que, à côté de l'expression études supérieures de sciences de l'éducation, le Guide de l'étudiant en sciences pédagogiques emploie aussi études en sciences de l'éducation ${ }^{5}$.

Toujours d'après le grand dictionnaire Robert, on dit cours de chimie, d'algèbre, de littérature, de droit civil, de musique, de danse, de physique, mais suivre un cours en Sorbonne, car il ne s'agit plus alors de la discipline étudiée, mais de l'université qu'on fréquente. Par ailleurs, en ce qui touche aux grades, on trouve certes diplôme de philosophie (grand dictionnaire Robert), licence et maîtrise de sciences de l'éducation ${ }^{6}$, maîtrise d'informatique ${ }^{7}$, baccalauréat de technicien de l'informatique ${ }^{8}$, brevet professionnel d'informatique ${ }^{9}$, mais aussi baccalauréat de technicien en informatique ${ }^{10}$ et B.T. (pour brevet de technicien?) en mécanographie ${ }^{11 .}$

Au chapitre de la spécialisation des professions, nous avons limité notre enquête aux appellations formées avec les mots analyste, animateur, conseiller, courtier, dessinateur, entrepreneur, expert, ingénieur, inspecteur, moniteur, profes-

2. Paul Robert, Dictionnaire alphabétique et analogique de la langue française, Paris, Société du Nouveau Littré, 1964.

3. Grand Larousse encyclopédique, 10 vol., Paris, Larousse, 1970.

4. Relevé dans META, XVI, 1-2 (1971), p. 18.

5. Paul Juif et Fernand Dovero, Guide de l'étudiant en sciences pédagogiques, Paris, Presses Universitaires de France, 1972, p. 135 et 183.

6. Ibid., p. 187.

7. Guide des professions de l'informatique, Paris, Néret, 1971, p. 36.

8. Ibid., p. 19.

9. Ibid., p. 57

10. Ibid., p. 18

11. Ibid., p. 68 . 
seur, spécialiste et technicien. Il y avait lieu de croire, en effet, que cet échantillonnage de termes courants, bien que sommaire, permettrait de dégager des constantes valables pour l'ensemble des locutions dérivées de mots semblables.

Les appellations d'emploi constituées avec le vocable animateur utilisent soit la préposition de (animateur de radio ${ }^{12}$, animateur de guignol ou de marionnettes ${ }^{13}$ ), soit la forme adjectivale (animateur culturel, animateur rural ${ }^{14}$ ). De même, les seuls exemples que nous ayons de locutions où analyste figure en première position sont ceux de la Classification internationale type des professions (C.I.T.P.), analyste de professions, analyste de systèmes, dont les éléments sont unis par la préposition de, et celui du supplément du grand dictionnaire Robert, analyste-programmeur, où il y a simple juxtaposition.

Les locutions constituées avec le mot conseiller exigent généralement la préposition $d e$, d'après le Grand Larousse encyclopédique, qui donne conseiller du commerce extérieur, conseiller du travail et conseiller d'orientation. Cependant, comme on trouve aussi conseiller en économie domestique, dans la C.I.T.P., l'appellation de conseiller en bilinguisme, qu'utilise l'administration fédérale, semble donc justifiée.

Selon le grand dictionnaire Robert, lorsqu'ils sont employés au sens propre, les groupes constitués avec le mot courtier appellent tantôt en (courtier en vins, en librairie, en immeubles, en valeurs mobilières), tantôt de (courtier de marchandises, de publicité), mais au sens figuré, ils veulent obligatoirement la préposition de (courtier de chair humaine, de mariage, de galanterie).

Le mot dessinateur s'emploie soit avec un adjectif (dessinateur humoriste, industriel ${ }^{15}$, cartographe ${ }^{16}$, électricien ${ }^{17}$ ), soit avec la préposition de (dessinateur de publicité ${ }^{18}$, d'architecture ${ }^{19}$ ), soit avec en (dessinateur en chaudronnerie, en ferronnerie en serrurerie $\left.{ }^{20}\right)$. Certaines désignations sont même concurrentes, telles que dessinateur mécanicien, dessinateur en mécanique et dessinateur de fabrications mécaniques ${ }^{21}$.

Le cas du mot entrepreneur est particulièrement intéressant, car la formation des locutions qui en dérivent accuse une tendance paraissant aller à l'encontre du mouvement général. En effet, alors qu'on semble porté à remplacer souvent de par en ou, du moins, à employer indifféremment l'une ou l'autre préposition, nous avons l'impression que, dans le français dit universel, la forme entrepreneur en a été supplantée par sa concurrente. C'est en tout cas ce qui ressort d'une comparaison entre, d'une part, l'usage canadien, souvent archaïque, où seule la forme en est usitée, ainsi que l'article pertinent du grand dictionnaire Harrap ${ }^{22}$,

12. Dictionnaire encyclopédique Quillet, 8 vol., Paris, Librairie Aristide Quillet, 1968.

13. Dictionnaire des métiers et appellations d'emploi, Paris, Presses Universitaires de France, 1955.

14. Classification internationale type des professions, Genève, Bureau international du Travail, 1968.

15. Paul Robert, Dictionnaire alphabétique et analogique de la langue française.

16. Grand Larousse encyclopédique.

17. Dictionnaire des métiers et appellations d'emploi.

18. Paul Robert, Dictionnaire alphabétique et analogique de la langue française.

19. Dictionnaire des métiers et appellations d'emploi.

20. Ibid.

21. Ibid.

22. J. E. Mansion, Harrap's Standard French and English Dictionary, London, George G. Harrap and Co. Ltd., 1970. 
qui ne donne que des exemples comportant la jonction en, et, d'autre part, le grand dictionnaire Robert et le Dictionnaire des métiers et appellations d'emploi (D.M.A.E.), dont les très nombreux exemples - qu'il serait trop long de reprendre ici et auxquels nous renvoyons le lecteur - sont tous formés avec la préposition de, à l'exception d'entrepreneur-chevillard (D.M.A.E.), constitué par juxtaposition ${ }^{23}$.

Suivant le grand dictionnaire Robert et le D.M.A.E., les locutions dérivées du mot expert prennent soit l'adjectif (expert agricole, comptable, économique, fiscal, etc.), soit la préposition en (expert en écritures, en assurances, en tableaux, en sculptures, en livres anciens, etc.).

La plupart des groupes construits avec le terme ingénieur sont généralement articulés autour de la jonction de ou constitués par simple juxtaposition. Pourtant, la jonction en se rencontre aussi : ingénieur en appareillage électrique, en appareils de ventilation, en constructions navales, en mécanique, en organisation (D.M.A.E.); ingénieur en climatisation, en couleurs et peintures, en froid industriel, etc. (C.I.T.P.). Comme pour le mot dessinateur, certaines appellations sont ici aussi concurrentes, notamment ingénieur mécanicien et ingénieur en mécanique (D.M.A.E.).

Il semble que le mot inspecteur ne forme de locutions qu'avec la préposition de, du moins à en juger par les exemples qu'apportent le grand dictionnaire Robert (inspecteur des manufactures, du travail, des finances, des contributions, d'assurances, de la navigation, de police, etc.) et la C.I.T.P. (inspecteur des poids et mesures, des services de transport ferroviaires, etc.).

Pour moniteur, c'est aussi la forme de qui s'impose, comme en font foi les exemples relevés au grand dictionnaire Robert (moniteur d'aviation, de ski, d'éducation physique) et au D.M.A.E. (moniteur d'agriculture, de pilotage, etc.).

Les trois principales sources consultées ne mentionnent aucune appellation formée avec le mot professeur suivi de la préposition en. Ces sources sont le Grand Larousse encyclopédique, et surtout le grand dictionnaire Robert et le D.M.A.E., qui donnent chacun une dizaine d'exemples avec la jonction de. Toutefois, on peut lire, dans la liste alphabétique servant d'index à la C.I.T.P., professeur en art dentaire, en sciences politiques, en sciences sociales. Il importe pourtant de noter que ces désignations ne sont pas utilisées dans le corps de l'ouvrage.

Bien que le terme de spécialiste commande en général la préposition de, les exceptions avec en existent aussi : spécialistes en informatique (C.I.T.P.), spécialiste en appareillages électriques d'avions (D.M.A.E.).

Ainsi qu'en témoignent les nombreux exemples de la C.I.T.P., les appellations constituées à partir du mot technicien se construisent d'ordinaire avec de (technicien de chimie industrielle, de télévision, des télécommunications, etc.). mais l'apposition se rencontre également (technicien chimiste, électricien, méca-

23. L'expression entrepreneur en construction a été utilisée il y a un certain temps dans une nouvelle en provenance de l'agence France-Presse, reproduite par le quotidien le Devoir. Reste à savoir si c'est la rédaction du journal montréalais qui a corrigé la dépêche d'outreAtlantique, ou si la tournure est vraiment moins vieillie en France que ne paraissent l'indiquer nos deux sources de référence. 
nicien, etc.), de même que la jonction en (technicien en céramique, en chauffage, en éclairage, en micromécanique, en radar, en ventilation).

Le tableau récapitulatif qui suit (où les emplois les moins courants sont indiqués entre parenthèses) permet de noter, d'abord, que l'utilisation de la préposition de n'est exclue qu'exceptionnellement (dans le cas des mots étudiant et expert) et, en second lieu, de confirmer le jugement formulé provisoirement à propos du mot entrepreneur (tendance à l'indifférentiation entre de et en). Il est probable qu'une étude portant sur un échantillonnage plus étendu ne ferait que renforcer ces constatations.

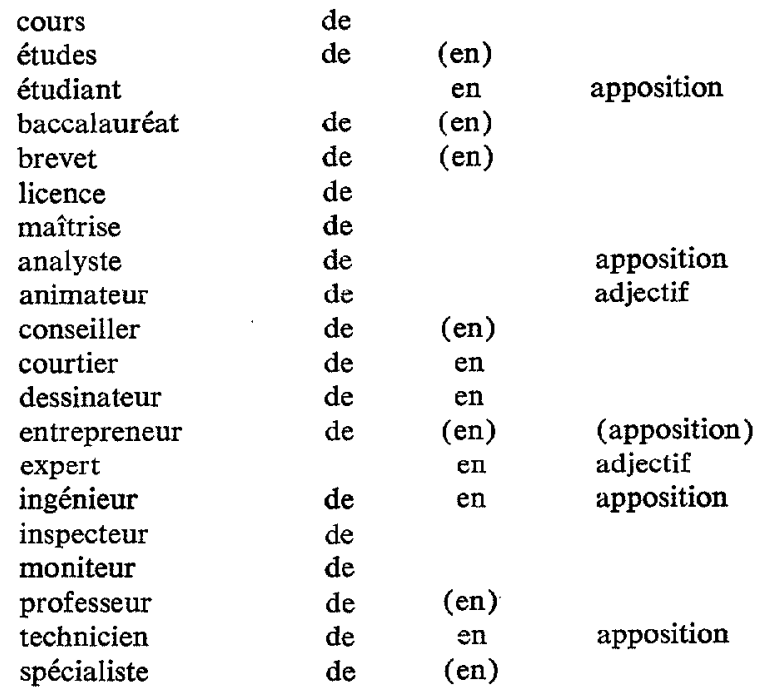

Bruno Couture

\section{AU PILORI}

\section{PAS SÉRIEUX, S'ABSTENIR}

\section{Conseilleur De Rehabilitation}

Le Service Pour La Rehabilitation Vocationnelle Du Ministere De La Famille et $\mathrm{Du}$ Bien-Etre Social, demand un assistant social professionnel pour entreprendre la rehabilitation vocationnelle des personnes physiquement et mentlement handicapes, à Cornwall. Les candidats elus etablirent la nature de les facteurs medicaux, sociaux et vocationnel, lesquels sont motif de dependance, et developperent et acheverent un traitement et un programme d'education pour retablir la personne a un emploi profitable.

Qualifications : Il faut que les candidats soient licencies en M.S.W. ou en B.S.W. et aient un interet a la rehabilitation. La connaissance de la langue française est indispensable. Un licencie en lettre qui a de l'experience serait considere pour un traitement inferieur.

La RÉDACTION 